\title{
Pulmonary metastasis from a World Health Organization grade I intracranial parasagittal meningioma: a case report
}

Peter YM Woo *, MB, BS, FRCS, Remy SL Hung, MB, BS, MRCS, Saori Takemura, MB, ChB, KY Chan, MB, ChB, FRCS, John CK Kwok, MB, ChB, FRCS

Department of Neurosurgery, Kwong Wah Hospital, Yaumatei, Hong Kong

*Corresponding author: wym307@ha.org.hk

Hong Kong Med J 2019;25:326-8

https://doi.org/10.12809/hkmj177059

\section{Case report}

A 37-year-old woman presented to our neurosurgical centre in January 2003 with a 2-month history of progressive blurred vision and was found to have papilloedema. Magnetic resonance imaging (MRI) scan of the brain revealed a large left frontal parasagittal extra-axial dural-based tumour with homogenous gadolinium contrast-enhancement (6.3 $\mathrm{cm} \times 4.3 \mathrm{~cm} \times 3.7 \mathrm{~cm}$ ) and Sindou grade II invasion (ie, into the lateral recess) into the junction of the anterior-to-middle third superior sagittal sinus (SSS). The patient underwent preoperative polyvinyl alcohol particle catheter tumour embolisation and a subsequent craniotomy was performed for Simpson's grade III excision (macroscopic complete excision without resection of the tumour's extradural extension into the SSS). The histological diagnosis was a World Health Organization (WHO) grade I meningothelial meningioma with a Ki-67 proliferation index of 5\%. A 2-year surveillance MRI scan (Fig 1a) revealed an asymptomatic local recurrence with further invasion into the SSS (Sindou grade IV, ie, involvement of the roof and lateral wall). The patient was asymptomatic and reluctant to undergo further treatment, opting for regular observation of the lesion. A new MRI scan performed 11 years after the first operation revealed interval tumour growth with complete occlusion of the SSS (Sindou grade V) that was confirmed with MR venography (Fig $1 \mathrm{~b}$ and $\mathrm{c}$ ). A second craniotomy was performed in October 2014, 11 years after the first, but only subtotal excision could be achieved because of dense tumour adhesions to a large posterior frontal cortical draining vein. The histology remained that of a WHO grade I meningioma.

From a preoperative chest X-ray, performed in preparation for the patient's second craniotomy, a new opacity in the right lower lobe was incidentally discovered. Computed tomography scan of the thorax revealed a single right lower lobe lung nodule $(2.4 \mathrm{~cm} \times 2.8 \mathrm{~cm} \times 2.3 \mathrm{~cm})$ with a well-defined border and vivid homogenous contrast enhancement (Fig $1 \mathrm{~d}$ and e). Video-assisted thoracoscopic wedge resection of the right lower lobe was performed 8 weeks after the craniotomy with gross total excision achieved. The final pathological diagnosis was a metastatic WHO grade I meningioma with a Ki-67 proliferation index of $1 \%$ and clear margins (Fig 1f).

In view of residual intracranial disease, the patient underwent adjuvant fractionated radiotherapy $(50.4 \mathrm{~Gy})$. At 2 years after the second craniotomy, surveillance MRI brain scans and chest $\mathrm{X}$-rays showed no detectable tumour.

\section{Discussion}

Meningiomas are the most frequently diagnosed primary brain tumour in adults, accounting for $13 \%$ to $26 \%$ of all lesions. ${ }^{1}$ The population incidence is estimated to be four to six per 100000 with a female:male ratio of $2: 1$. Despite this high prevalence, distant (extracranial) metastasis is extremely rare with fewer than 120 cases reported. $^{1}$

The grading of meningiomas is principally determined by light microscopy of haematoxylineosin sections in accordance with WHO criteria. Grade I intracranial meningiomas comprise $80 \%$ of tumours and are generally considered benign, slow-growing lesions that have no demonstrable malignant behaviour such as distant metastasis. However, contrary to this belief, one third of meningiomas with distant metastases originate from grade I tumours with $31 \%$ identified incidentally. ${ }^{1}$ In contrast, grade III lesions, which demonstrate overt aggressive behaviour, represent only $1 \%$ of meningiomas and account for $40 \%$ of documented metastases. ${ }^{1}$ The true incidence of metastatic meningiomas is unknown, but given the frequent occurrence of grade I tumours, that metastatic lesions are often asymptomatic and that routine whole-body imaging is seldom performed, the stated figure of $0.1 \%$ is likely an underestimation. ${ }^{1}$ In our case, the interval between primary resection and metastasis detection was 11 years, considerably longer than the cited median duration of 58 months (range, 4 months to 15 years), reflecting the slowgrowing nature of grade I tumours. ${ }^{1}$

Three quarters of metastatic WHO grade I meningiomas involve a single organ, primarily 


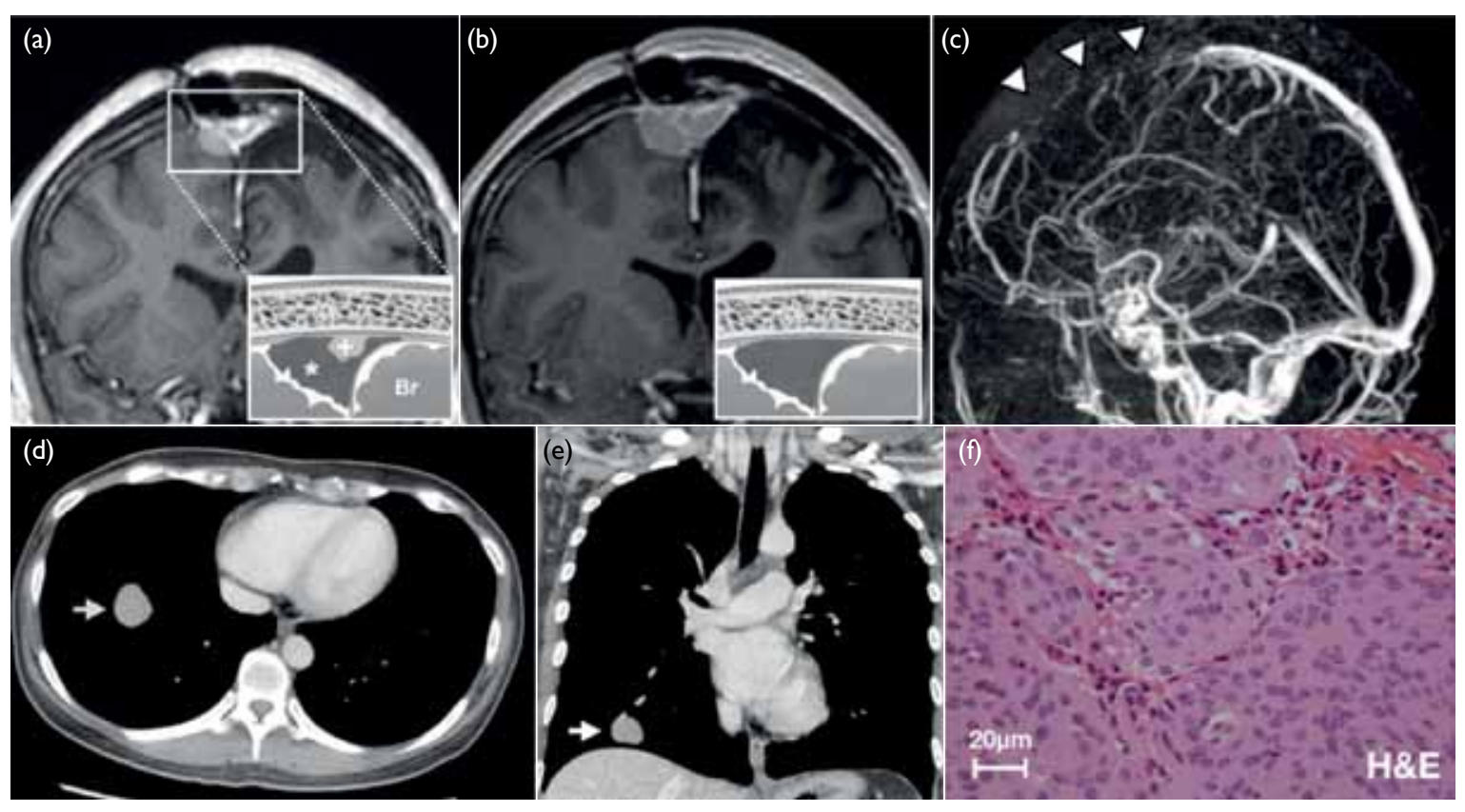

FIG. (a) Coronal contrast-enhanced magnetic resonance imaging (MRI) brain scan 2 years after the first craniotomy showing a recurrent parasagittal meningioma (white asterisk) with Sindou grade IV infiltration (inset: schematic; $\mathrm{Br}$, brain) of the superior sagittal sinus (SSS) (white cross). (b) Coronal contrast-enhanced MRI brain scan II years after the first craniotomy showing interval tumour growth with Sindou grade V complete occlusion of the SSS (inset: schematic). (c) Left sagittal view contrast-enhanced MR venogram confirming complete anterior SSS occlusion (white arrowheads). (d) Axial and (e) coronal contrast-enhanced computed tomography scans of the thorax showing a single circumscribed right lower lobe lung lesion with homogenous enhancement (white arrows). (f) Haematoxylin-and-eosin stain photomicrograph of the pulmonary tumour showing a meningotheliomatous meningioma with uniform tumour cells arranged in tight whorls

the lung $(42 \%)$ followed by the spine (12\%), bone $(10 \%)$, liver (10\%), and cervical lymph nodes $(10 \%){ }^{1}$ Although conventional histological studies such as the Ki-67 proliferation index have failed to identify a subgroup of meningiomas predisposed to metastasis, loss of heterozygosity of $1 p, 9 p, 14 q$ and $22 \mathrm{q}$ may be characteristic of these lesions. ${ }^{2}$ Clinical risk factors for metastasis include repeated surgery, local recurrence and invasion of the dural venous sinuses. ${ }^{1}$ The non-collapsible and valve-less nature of the dural venous sinuses, such as the SSS, may permit seeding of tumour cells into the internal jugular vein and subsequently into the pulmonary microcirculation, an indication that tumour location is pivotal in determining haematogenous metastasis. ${ }^{1}$ Parasagittal meningiomas, comprising $20 \%$ to $34 \%$ of lesions, are perhaps most susceptible because of their propensity to invade the SSS, technically hindering their complete resection. ${ }^{3,4}$ Our case illustrates the importance of treating the SSS infiltrating portion of these tumours, but there is little consensus on the appropriate management strategy. When the posterior SSS is patent, prohibiting its ligation and excision, some neurosurgeons prefer subtotal resection followed by adjuvant radiosurgery or radiotherapy. ${ }^{3}$ Others advocate the more technically demanding surgical approach of gross total resection with sinus reconstruction, to spare the patient the long-term adverse effects of irradiation. ${ }^{4}$ Both strategies offer comparable tumour control rates although multimodality treatment may be associated with fewer procedure-related complications. ${ }^{3}$

Bronchogenic carcinoma is the most important differential diagnosis to exclude in patients with pulmonary meningioma metastasis, but it is difficult to distinguish on computed tomography imaging. Meningioma metastases are usually single, noncalcified well-circumscribed lesions that may display strong homogenous contrast-enhancement. ${ }^{1}$ ${ }^{111}$ Indium-octreotide imaging is useful in identifying meningiomas, exhibitingaviduptake, butits restricted availability limits its use. ${ }^{5}$ Excision of the pulmonary lesion is recommended to establish the diagnosis and in some instances the meningioma metastasis may manifest a more aggressive grading than the primary lesion warranting adjuvant radiotherapy. ${ }^{5}$ When multiple disseminated metastases preclude surgical excision, systemic treatments such as octreotide acetate or bevacizumab, an anti-angiogenic therapy directed against vascular-endothelial growth factor have shown some promise for tumour control. ${ }^{5}$ However, in a case series of patients with recurrent 
meningioma refractory to surgery, radiotherapy and chemotherapy, pulmonary metastasis was identified as an unfavourable prognostic factor for overall survival. ${ }^{5}$

Distant metastasis from a WHO grade I meningioma is a rare phenomenon and can occur more than a decade after the initial diagnosis of the primary tumour. This case demonstrates that, regardless of grading and especially when the patient is young, meningiomas that infiltrate the dural venous sinuses require proactive management, either by adjuvant irradiation or by gross total resection.

\section{Author contributions}

All authors had full access to the data, contributed to the study, approved the final version for publication, and take responsibility for its accuracy and integrity.

Concept of study: PYM Woo, RSL Hung.

Acquisition of data: PYM Woo, RSL Hung.

Analysis of data: PYM Woo, RSL Hung, S Takemura.

Drafting of the article: PYM Woo, RSL Hung, S Takemura.

Critical revision for important intellectual content: All authors.

\section{Conflicts of interest}

All authors have disclosed no conflicts of interest.

\section{Funding/support}

This research received no specific grant from any funding agency in the public, commercial, or not-for-profit sectors.

\section{Ethics approval}

Ethics committee approval was waived because this is a case report and no personal identifying information was disclosed. A signed patient consent statement was obtained.

\section{References}

1. Surov A, Gottschling S, Bolz J, et al. Distant metastases in meningioma: an underestimated problem. J Neurooncol 2013;112:323-7.

2. Gladin CR, Salsano E, Menghi F, et al. Loss of heterozygosity studies in extracranial metastatic meningiomas. J Neurooncol 2007;85:81-5.

3. Gatterbauer B, Gevsek S, Höftberger R, et al. Multimodal treatment of parasagittal meningiomas: a single-center experience. J Neurosurg 2017;127:1249-56.

4. Ricci A, Di Vitantonio H, De Paulis D, et al. Parasagittal meningiomas: our surgical experience and the reconstruction technique of the superior sagittal sinus. Surg Neurol Int 2017;8:1.

5. Alexandru D, Glantz MJ, Kim L, Chamberlain MC, Bota DA. Pulmonary metastases in patients with recurrent, treatment-resistant meningioma: prognosis and identification by 111Indium-octreotide imaging. Cancer 2011;117:4506-11. 